\title{
Interventional Spine Considerations for Radiosensitivity in a Patient With Ligase IV Syndrome
}

\author{
David J. Kohns, DO \\ Mahmood Gharib, MD
}

\begin{abstract}
Background: Patient selection plays a critical role in any interventional pain practice. Rare conditions may not always have clearly established guidelines for interventional pain procedures. Ligase IV (LIG4) syndrome is a rare inherited condition with a wide variety of features, including radiosensitivity..
Case Report: A 36-year-old woman with a known history of LIG4 syndrome presented with an acute on chronic left L5 and S1 radiculopathy from a large L5-S1 central disc herniation. In an effort to minimize radiation expo- sure, an ultrasound (US)-guided caudal epidural steroid injection was used to help manage her radicular leg pain.
Conclusion: Interventional spine providers should be aware of rare radiosensitive conditions and strive to offer ap- propriate treatment alternatives. While limitations do exist, emerging US-guide spine procedures hold a distinct advantage for patients with radiosensitivities.

Key words: Epidural steroid injection, Ligase IV syndrome, radiculopathy, radiology, radiosensitivity, ultrasound 


\section{BACKGROUND}

LIG4 syndrome is a rare autosomal recessive immunodeficiency where a fault in DNA ligase IV impacts the repair of DNA double-strand breaks. While DNA is under constant threat of damage from reactive oxygen species and ionizing radiation, normally mechanisms are in place to recognize and repair these errors to maintain genomic integrity. These errors affect phases of cellular division during meiosis and may result in carcinogenesis or cellular death through apoptosis. DNA repair mechanisms also play a role in the production of diverse T- and B-lymphocytes in providing adaptive immunity (1). For patients with radiosensitivity who present with radicular back pain, reducing exposure to potentially damaging ionizing radiation must be considered prior to any interventional spinal procedure (2). Unlike x-ray, there is no ionizing radiation with use of ultrasound (US) for image-guided procedures. Distinct advantages and disadvantages should be considered with the use of US for spine procedures.

\section{CASE DESCRIPTION}

A 36-year-old woman presented to a tertiary spine care program with acute on chronic back and left leg pain with weakness and positive neural tension signs. She had a rather complex medical history that included known history of LIG4 syndrome with physical features of short stature, developmental delays (primarily speech delay and learning difficulties), diabetes mellitus type 2, premature ovarian failure, parotid tumor status post resection, and hepatocellular carcinoma status post resection with subsequent pancytopenia. She underwent whole exome sequencing in 2017 that showed 2 pathogenic changes, a maternally inherited c. 2440 C $>T\left(p . R 814^{*}\right)$ mutation and a paternally inherited c.613delT (p.S205Lfs*29) mutation in her LIG4 genes, consistent with a diagnosis of DNA ligase IV deficiency (LIG4 syndrome).

The patient experienced chronic back pain for about 6 months prior to being referred to a physical medicine and rehabilitation spine clinic. At that time she had reported no recent major infections, bruising/bleeding, melena, hematemesis, lymphadenopathy, or fevers. She reported that her back pain initially started while in high school while participating in gymnastics. She was later involved in a motor vehicle accident in 2005 with intermittent back pain over the years. She had no prior physical therapy, injections, or surgery to address her back pain. Over the previous 2 to 3 months, she developed worsening back and left leg pain.
Pertinent findings on neuromuscular examination showed strength deficits with left great toe extension and plantar flexion at 4 out of 5 on manual muscle testing. Her deep tendon reflexes were diminished at the left Achilles. The slump test was positive for left lower extremity neural tension. There was lower midline spinal tenderness. Her lumbar flexion was limited to $50 \%$ by neural tension pain. There were no findings to suggest any upper motor neuron lesion findings. A review of recent lumbar magnetic resonance imaging (MRI) showed a large central L5-S1 disc herniation.

Based on her reported symptoms, physical examination, and MRI findings, her condition was consistent with a left L5 and S1 radiculopathy from a large L5-S1 central disc herniation. Due to radiosensitivity, her interventional treatment options were limited. Oncology expressed that their priority would be to minimize the patient's radiation exposure to the lowest possible level, and favored image-guided techniques that would avoid ionizing radiation.

With consideration of the patient's chief complaint and underlying radiosensitive LIG4 syndrome, it was decided to proceed with a multimodal approach to the management of her left L5-S1 radiculopathy. The patient was referred to physical therapy for a directional preference exercise program in order to address her radicular back pain. For neuropathic pain, a trial of gabapentin was ordered. It was recommended she use a limited amount of over-the-counter nonsteroidal antiinflammatories for additional analgesia. As she failed to adequately respond to these initial measures after 2 weeks, an US-guided caudal epidural steroid injection (ESI) targeting her lower lumbosacral radiculopathy pain was offered.

After obtaining consent, the patient elected to proceed with an US-guided caudal ESI. An 18-5 MHz linear array transducer (Affinity System; Philips Healthcare, Bothell, WA) was used to identify the sacral hiatus and sacra conua. After local anesthetic with $1 \%$ lidocaine, a 22-gauge x 3 1/8-inch (80-mm) EchoBlock needle (Havel's Inc., Cincinnati, $\mathrm{OH}$ ) was advanced in a caudalto-cranial direction utilizing an in-plane approach with the transducer to the long axis under continuous US guidance. The tip of the needle was visualized throughout the procedure until it was felt to pass just deep to the sacrococcygeal ligament (Figs. 1). After negative aspiration, a 6-mL treatment solution consisting of $1 \mathrm{~mL}$ of methylprednisolone acetate $40 \mathrm{mg} / \mathrm{mL}, 2 \mathrm{~mL}$ of $1 \%$ lidocaine, and $3 \mathrm{~mL}$ of $0.9 \%$ preservative-free normal 

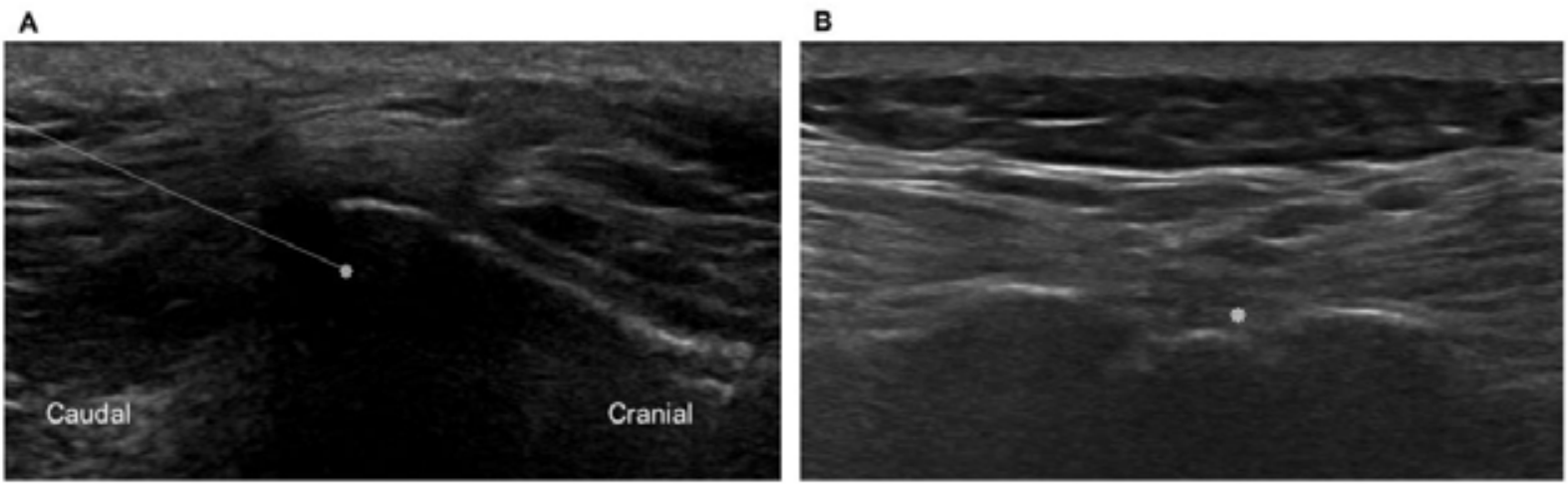

Fig. 1. (A) In-plane, long-axis trajectory view of caudal epidural needle placement within the sacral hiatus. Dashed line and * = needle position. (B) Out-of-plane, short-axis view to reconfirm the epidural needle position. ${ }^{*}=$ needle tip position.

saline was injected slowly into the affected area. At follow-up, the patient reported about $80 \%$ relief from her leg pain the day of the injection. This relief tapered down to about $50 \%$ at 10 weeks when she requested to repeat the US-guided caudal ESI.

\section{DISCUSSION}

As a rare immunodeficiency, there are only 28 reported cases of LIG4 syndrome globally. The diagnostic process for LIG4 syndrome starts with clinical suspicion based on neurodevelopmental delays, recurrent infections, and radiosensitivity. The physical presentation of LIG4 syndrome includes a wide spectrum of developmental delays from minimal to profound. There is marked microcephaly, commonly with Seckel syndrome (bird-like facial features with beak-like nose, receding forehead, and micrognathia). Bony changes are also common and may include hypoplasia, syndactyly, polydactyly, and congenital hip dysplasia. There have also been reported reproductive and dermatologic changes. Genetic testing is used to identify a deficiency of Lig4 (1).

Due to associated immunodeficiencies, patients with LIG4 syndrome have marked T- and B-lymphocytopenia, predisposing them to bacterial, viral, and fungal infections. Patients with LIG4 syndrome can present with a wide range of immunodeficiency from no evidence to severe combined immunodeficiency (SCID) (3).

The primary treatment for LIG4 syndrome includes hematopoietic stem cell transplant to address immunodeficiency. In addition, long-term antibiotic, antiviral, and antifungal mediations are used for infection prophylactics.
Table 1: Conditions with radiosensitivity

\begin{tabular}{|c|}
\hline Ataxia-telangiectasia (A-T) \\
\hline Nijmegen breakage syndrome (NBS) \\
\hline Fanconi anemia (FA) \\
\hline DNA ligase IV deficiency (LIG4) \\
\hline Bloom's syndrome \\
\hline$B R C A 1$ or $B R C A 2$ \\
\hline
\end{tabular}

\section{Radiosensitivity}

The potential for adverse reactions from harnessed radiation has been known nearly as long as its discovery. While radiation safety should be a concern for all patients, there are populations that have been recognized as being radiosensitive. Up to 40 DNA repair disorders have been identified with variable adverse reactions to radiation exposure (Table 1). These pose both diagnostic and therapeutic challenges to clinicians. Though rare, ataxia-telangiectasia (A-T), Nijmegen breakage syndrome (NBS), Fanconi anemia (FA), and DNA ligase IV deficiency are a few of the rare inherited conditions associated with radiation sensitivity. While the above homozygotic disorders may have a more profound reaction to ionized radiation, there are also a number of heterozygotic disorders (BRACA1 or BRAC2) that may also have a higher threshold of radiosensitivity. Based on clinical suspicion, genetic testing is the standard approach to identifying many of these conditions $(1,4,5)$.

Once identified, patients with these DNA repair disorders should avoid, or minimize, ionizing radiation from diagnostic testing that includes $x$-ray and computed tomography $(\mathrm{CT})$. Unfortunately, the failure to repair 
DNA double-strand breaks predisposes these patients to developing various cancers. This presents a conundrum for the radiation oncologist, as radiosensitivity reactions are a potential risk for iatrogenic injury from radiation therapy used in the treatment of many cancers. Based on these risks, dosing protocols may have to be adjusted, or radiation therapy may be contraindicated altogether (4).

For interventional spine physicians, the use of fluoroscopic imaging is the standard for guiding various types of ESI in the management of radicular back pain (6). It has been reported that the mean radiation exposure from a fluoroscopy-guided lumbar transforaminal epidural steroid injection (TFESI) is $0.24 \mathrm{mSv} \pm 0.22$. By comparison, the typical chest $\mathrm{x}$-ray exposure is about 0.1 $\mathrm{mSv}$ (7). While this level of radiation exposure pales in comparison to the potential radiation exposure from CT or radiation therapy, it still poses a potential risk for patients with radiosensitivities. Whenever possible, minimizing or eliminating the need for radiation exposure is recommended for patients with one of these rare DNA repair disorders (4).

As an emerging technology in musculoskeletal medicine, US has now become commonplace for peripheral joint injection interventions. Benefits of musculoskeletal US include the ability to provide high-resolution, realtime imaging to guide procedures without the use of radiation exposure (8). US has potential benefits that include high-resolution linear array probes, tissue harmonic imaging, 2-dimensional matrix probe technology, extended field-of-view imaging, and power Doppler (9). Still, US has been less utilized for axial spine procedures. US-guided spinal procedures have a disadvantage in that the clinician cannot monitor the depth of the needle placement beyond osseous structures due to the high reflectivity of the acoustic interface. Visualizing contrast medium spread is also less effective with US. Both of these are distinct advantages of fluoroscopy-guided procedures $(9,10)$.

Caudal ESIs have been done for years based solely on palpation of the sacral hiatus as an anatomical landmark. Fluoroscopic x-ray later emerged as the gold standard for caudal access (6). Recently, the use of US has been suggested for the guidance of caudal ESI.
Hazra et al (11) performed a randomized-controlled study assigning 50 patients with radicular pain to receive either fluoroscopy- or US-guided caudal ESI. There were no statistical differences in their scores on the Oswestry Disability Index (ODI) or Verbal Numeric Pain Scale, and less time was required for needle placement in the US group. Similar findings by Park et al (12) with 110 patients support these findings with a success rate of the US-guided needle placement of $85 \%$ confirmed under fluoroscopic contrast images. In addition to caudal approaches, Yang et al (13) compared the accuracy, effect on pain relief, and safety of US- vs fluoroscopy-guided lumbar TFESI. They reported no significant difference in pain relief between groups and no serious complication in either the US or fluoroscopic transforaminal approach. A systemic review and meta-analysis by Liu et al (14) in 2016 found that both the fluoroscopy-guided TFESI and caudal approaches were similarly effective in reducing pain and improving functional scores in the management of lumbosacral radicular pain.

Given the comparable efficacy and relative safety demonstrated in both fluoroscopy-guided lumbar TFESI and caudal ESI approaches, with similar findings from fluoroscopic and US guidance in performing caudal ESI, further head-to-head research is needed to compare US-guided caudal to fluoroscopy-guided lumbar transforaminal approaches for management of patients with lumbosacral radicular pain.

\section{CONCLUSION}

Interventional spine physicians should be aware of patients with rare radiosensitivities and be prepared to consider alternative treatment approaches. US-guided caudal ESI procedures are a reasonable, and likely preferred, option in the management of radicular back pain for patients with radiosensitivity. While having distinct advantages and disadvantages, US-guided procedures for the spine continue to emerge.

This case report conforms to all CARE guidelines and reports the required information accordingly (see Supplementary Checklist). 


\section{REFERENCES}

1. Altmann T, Gennery AR. DNA ligase IV syndrome; a review. Orphanet J Rare Dis 2016; 11:137.

2. Chistiakov DA. Ligase IV syndrome. Adv Exp Med Biol 2010; 685:175-185.

3. O'Driscoll M, Cerosaletti KM, Girard PM, et al. DNA ligase IV mutations identified in patients exhibiting developmental delay and immunodeficiency. Mol Cell 2001; 8:1175-1185.

4. Pollard JM, Gatti RA. Clinical radiation sensitivity with DNA repair disorders: An overview. Int J Radiat Oncol Biol Phys 2009, 74:1323-1331. doi:10.1016/j.jirobp.2009.02.057.

5. Gruhn B, Seidel J, Zintl F, et al. Successful bone marrow transplantation in a patient with DNA ligase IV deficiency and bone marrow failure. Orphanet J Rare Dis 2007; 2:1-8. doi:10.1186/17501172-2-5.

6. Stitz MY, Sommer HM. Accuracy of blind versus fluoroscopically guided caudal epidural injection. Spine (Phila Pa 1976) 1999, 24:1371-1376

7. Dietrich TJ, Peterson CK, Zeimpekis KG, Bensler S, Sutter R, Pfirrmann CWA. Fluoroscopy-guided versus CT-guided lumbar steroid injections: Comparison of radiation exposure and outcomes. Radiology 2019; 290:752-759.

8. Smith J, Finnoff JT. Diagnostic and interventional musculoskeletal ultrasound: Part 1. Fundamentals. PM R 2009; 1:64-75

9. Park Y, Lee JH, Park KD, Ahn JK, Park J, Jee H. Ultrasound-guid- ed vs. fluoroscopy-guided caudal epidural steroid injection for the treatment of unilateral lower lumbar radicular pain: A prospective, randomized, single-blind clinical study. Am J Phys Med Rehabil 2013; 92:575-586.

10. Smith J, Finnoff JT. Diagnostic and interventional musculoskeletal ultrasound: Part 2. Fundamentals. PM R 2009; 1:162-177.

11. Hazra AK, Bhattacharya D, Mukherjee S, Ghosh S, Mitra M, Mandal M. Ultrasound versus fluoroscopy-guided caudal epidural steroid injection for the treatment of chronic low back pain with radiculopathy: A randomised, controlled clinical trial. Indian J Anaesth 2016; 60:388-392.

12. Park KD, Kim TK, Lee WY, Ahn J, Koh SH, Park Y. Ultrasound-guided versus fluoroscopy-guided caudal epidural steroid injection for the treatment of unilateral lower lumbar radicular pain: Case-controlled, retrospective, comparative study. Medicine (Baltimore) 2015; 94:e2261.

13. Yang G, Liu J, Ma L, et al. Ultrasound-guided versus fluoroscopycontrolled lumbar transforaminal epidural injections: A prospective randomized clinical trial. Clin J Pain 2016; 32:103-108.

14. Liu J, Zhou H, Lu L, et al. The effectiveness of transforaminal versus caudal routes for epidural steroid injections in managing lumbosacral radicular pain: A systematic review and meta-analysis. Medicine (Baltimore) 2016; 95:e3373. 
